\title{
A Scalable and Distributed Mechanism for DNA Databases by Aggregate Queries
}

\author{
H Santhi, Gayathri P, Gopichand G, Venkata Vinod Kumar N, Sailaja G
}

\begin{abstract}
The matter of sharing unique individual genomic grouping courses of action without giving the security of their data to help vast scale biomedical research ventures. Regardless, extends the results in different types. This approach is demonstrated powerful in keeping up the protection requirement against antagonistic server. We present a cryptographic security for questions that permits playing out most widely recognized DNA based personality. The limit is more affordable than figuring in current dispersed registering assessing plans. This point is spurred by the way that capacity is less expensive than calculation in current distributed computing evaluating plans. In addition, encoding the information makes it workable for us to deal with more extravagant arrangement of the inquiries the coordinating between the inquiry and grouping of the database, including:
\end{abstract}

(1) A certain is the quantity that matches between question images and a succession;

(2) Consistent OR matches where a question image is permitted to coordinate a subset of the letters in order along these lines making it conceivable to deal with (as an uncommon case) a "not equivalent to" necessity for an inquiry image ("not a $\left.G^{\prime \prime}\right)$;

(3) Bolster for the expanded letter set of nucleotide base codes that envelops ambiguities in DNA groupings;

(4) Inquiries that determine the quantity of events of every sort of image in the predetermined arrangement positions.

(5) A begin question whose answer is "yes" if the quantity of matches surpasses a question indicated edge.

(6) All inquiry composes we can conceal appropriate responses from the unscrambling server, with the goal that just the customer takes in the appropriate response.

(7) The customer deterministically adapts just the question's answer, with the exception of inquiry compose (v) where we measure the (simple little) factual spillage to customer of real check.

Index Terms-DNA Databases, Cloud Security, Secure Outsourcing

\section{INTRODUCTION}

DNA is the medium of deep rooted stockpiling and transmission of hereditary data for all contemporary living creatures. Human DNA information is private and delicate individual data. Be that as it may, such information is basic, for instance, conclusion of attitude to execute particular infection, tranquilize hypersensitivity, or forecast of accomplishment rate in light of a particular treatment. Giving

Revised Version Manuscript Received on April 12, 2019.

H Santhi, School of Computer Science and Engineering, Vellore Institute of Technology, Vellore, T.N, India

Gayathri P, School of Computer Science and Engineering, Vellore Institute of Technology, Vellore, T.N, India

Gopichand G, School of Computer Science and Engineering, Vellore Institute of Technology, Vellore, T.N, India

Venkata Vinod Kumar N, Department of CSE, Annamacharya Institute of Technology and Sciences, Tirupati, A.P, India

Sailaja G, Department of CSE, SV Engineering College for Women, Tirupati, A.P, India an openly accessible DNA database for encouraging exploration in this field is for the most part stood up to by protection concerns.

Now a days, the substantial calculation and limit of cloud administrations empowers pragmatic facilitating and sending of DNA databases and effective preparing of genomic arrangements, for example, doing grouping examination, flawless and estimated succession seek and various tests (conclusion, character, family line). The missing security layer that jellies the protection of people reports and doles out the heap of inquiry handling to the cloud. Though anoymization strategies, for example, de-recognizable proof [2], information expansion [3], or database apportioning [4] settle this issue mostly, they are not adequate in light of the fact that much of the time, re-ID of people is conceivable [5]. It takes after that the DNA information must secured, not only unlinked from the relating people.

We manage system proposed in [1], in that the DNA reports originating from couple of healing centers are encoded and keep the information at an information stockpiling site, and biomedical analysts can submit total tallying inquiries to this site. Checking inquiries are especially intriguing for factual investigation.

\section{PROBLEM DEFINITION AND FRAMEWORK}

This proposed construction gives another strategy that tends to a bigger arrangement of issues and gives a quicker question answer time than procedure presented in [1]. Our methodology depends on the current estimating plans of many cloud administrations merchants, stockpiling is less expensive than figuring. Subsequently, we support stockpiling through registering assets to enhance cost. Besides, from a client encounter perspective, answer time is the most substantial marker of execution; henceforth it is normal to go for lessening it. Our technique builds the best in class at both the reasonable level and the execution level.

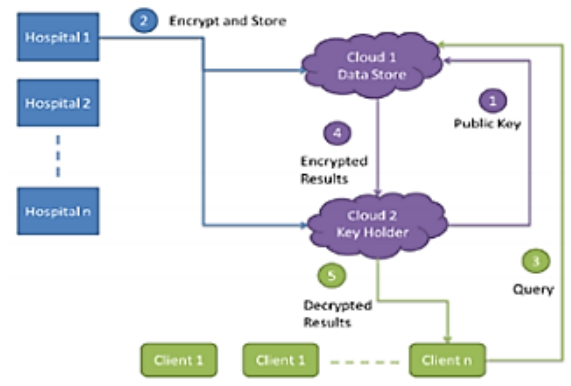

Fig.1. System Architecture

Published By:

Blue Eyes Intelligence Engineering

\& Sciences Publication 
We consider a structure like made out of a few healing facilities; a few customers speaking to biomedical scientists and two non plotting servers. In Fig1 we have two servers i.e. Cloud1 and Cloud2 to emphasize the system that can be conveyed in a cloud situation:

- First cloud stores the information, where all the DNA records are encoded and secured. Second cloud is trusted and gathers the information and holds the private and public key by using homomorphic encryption method. Stage 1 in general large key sent to alternate gatherings

- Second cloud is used as an separating prophet and it imparts security relationship to the clients to send the results securely.

- Here, People get a general key with a specific end goal to encode beneficiary DNA records and exchange them to first cloud (stage 2).

- Customer speaking to biomedical scientist presents question to first cloud (stage 3). The cloud checks the request over encoded records and sends the result to second cloud (stage 4).First cloud is required to permit the results of particular records before sending them out. The change of anchors of records for a circumstance of the demand of records that can be associated with a few ensured data. At long last the customer gets from second cloud, The unscrambled tally of matches (stage 5) through a protected channel (fabricated because of the security affiliation set up at stage 1 ).

- Second Cloud may help the information encryption at the information proprietors (the doctor's facilities) through pre-scrambling a wide number of characteristics for encoding of each letter in letter set is exchanging with the information proprietors.

\section{ANALAYTICAL RESULTS \& DISCUSSIONS}

M. Kantarcioglu, W. Jiang proposed cryptographic method to manage secure offer and request genomic groupings [1]. In this paper, creators present a novel cryptographic structure that empowers associations to help genomic information mining without uncovering the crude genomic arrangements. Associations contribute scrambled genomic succession records into a brought together store, where the manager can perform inquiries, for example, recurrence tallies, without decoding the information. They assess the effectiveness of their system with existing databases of single nucleotide polymorphism (SNP) arrangements and exhibit that the time expected to finish check inquiries is doable for certifiable applications. They additionally demonstrate that guess methodologies can be connected to fundamentally accelerate inquiry execution times with insignificant misfortune in exactness. The structure can be actualized over existing data and system advancements in biomedical conditions.

L. Sweeney, B. Malin exhibited how to secure genomic information security in a circulated arrange: utilizing trail re recognizable proof to assess and outline obscurity insurance frameworks [2]. This paper, creators examine the disintegration of security at the point when genomic information, either pseudonymous or information accepted to be mysterious, are discharged into a dispersed medicinal services condition. A few calculations are presented, on the

whole called RE Identification of Data in Trails, which interface genomic data to named people in freely accessible records by utilizing special highlights in quiet area visit designs. Algorithmic verifications of re recognizable proof are produced and we illustrate, with probes genuine information, that powerlessness to re-distinguishing proof is neither paltry nor the consequence of peculiar detached events. Creators suggest that such methods can be connected as framework trial of security assurance capacities.

M. Blanton, Y. Zhang, and E. Aguiar proposed an review of issues and late advancements in distributed computing and capacity security [3].The late quick development in the accessibility and prevalence of cloud administrations takes into consideration helpful on request remote stockpiling and calculation. Security and protection concerns, be that as it may, are among the best hindrances obstructing more extensive appropriation of cloud advancements. That is, notwithstanding new security dangers that rise with the selection of new cloud innovation, an absence of direct authority over one information calculation requests new methods for specialist organization's straightforwardness and responsibility. The objective of this section is to give a wide review of ongoing writing covering different parts of cloud security. Creators portray as of late found assaults on cloud suppliers and their countermeasures, and in addition assurance components that go for enhancing security and trustworthiness of customer's information and calculations. The points shrouded in this review incorporate validation, virtualization, accessibility, responsibility, and protection and honesty of remote stockpiling and calculation.

M. Jakobsson and P. Bohannon introduced a cryptographic approach to Privacy in Forensic DNA Database [4]. Makers think about get the chance to control for one class of such databases, criminological DNA databases, used to facilitate darken guilty parties against get-togethers of potential suspects - more often than not sentenced crooks. Our key perception is that for real criminological questions, the delicate data having a place with the objective individual is as of now accessible to the questioning operator as a blood test from wrongdoing scene. They indicate how criminological DNA database might be executed with the goal that just genuine questions are possible. Specifically, a man with boundless access to the database will be notable concentrate data about any individual except if the vital hereditary data for that individual is as of now known. They build up a general arrangement system, and demonstrate to actualize databases which handle certain instances of absent or off base DNA tests. This structure and systems are relevant to the general issue of scrambling data in light of incompletely known or mostly amend keys, and its security depends on standard cryptographic suspicions.

S. Katzenbeisser and F. Bruekers, exhibited security saving coordinating of DNA profiles [5].In this paper, creators present cryptographic protection improving conventions that permit playing out the most well-known DNA based character, paternity and parentage tests and consequently actualizing protection upgraded online ancestry 
administrations or research ventures. In the semi-legitimate aggressor demonstrate, the conventions ensure that no touchy data about the included DNA is uncovered, and are strong against regular types of estimation mistakes amid DNA sequencing. The conventions are commonsense and productive, both regarding correspondence and calculation multifaceted nature.

M. Aliasgari and M. Blanton, proposed secure redistributing of DNA looking by means of limited automata [6]. This work treats the issue of blunder flexible DNA looking through un mindful assessment of limited automata, where a customer has DNA succession, and specialist organization has an example that compares to a hereditary test. Mistake flexible looking is accomplished by speaking to the example as a limited robot and assessing it on the DNA succession (which is dealt with as the info), where security of both the example and the DNA grouping must be protected. Intuitive answers for this issue as of now exist, however can be a weight on the taking an interest parties. In this work creators propose procedures for secure outsourcing of careless assessment of limited automata to computational servers, to such an extent that the servers don't take in any data. Our procedures are pertinent to limited automata; however the improvements are customized to the setting of DNA seeking.

M. J. Atallah and J. Li, proposed secure outsourcing of succession correlations [7].Internet figuring innovations, similar to network processing, empower a feeble computational gadget associated with such a framework to be less constrained by its lacking neighborhood computational, stockpiling, and transmission capacity assets. Be that as it may, such a feeble computational gadget (PDA, smartcard, sensor, and so forth.) regularly can't benefit itself of the plenteous assets accessible on the system since its information is delicate. This propels the outline of methods for computational re-appropriating in a security safeguarding way, without uncovering the remote administrators whose computational power is being used either one's data or the consequence of the figuring. This paper states the secure re-appropriating for broadly relevant arrangement correlation issues and gives an effective convention for a client to safely outsource grouping correlations with two remote operators. The calculations done by the customer is immediate to proportion of the groupings, the computational expense and proportion of correspondence is done by external administrators and it is close to the time multifaceted nature of best count of dealing with the issue on a single machine.

Q. M. Malluhi, A. E. Nergiz, and C. Clifton, displayed refreshing examined databases [8].Authors acquaint activities with securely refresh a dissected database. The outcome is where the perspective of the server fulfills models, for example, k-obscurity or 1-assorted variety, however the customer can inquiry and change the first information. By uncovering information where conceivable, the server can perform esteem included administrations, for example, information examination impractical with completely encoded information, while as yet being not able damage protection limitations. Refresh is a key test with this model; local utilization of addition and erasure activities uncovers the real information to the server. This paper demonstrates how information can be securely embedded,

erased, and refreshed. The key thoughts are that information is embedded or refreshed into an encoded transitory table until the point that enough information is accessible to securely decode, and that delicate data of erased tuples is deserted to guarantee protection of both erased and undeleted people.

J. Winn, L. Sweeney, and A. Abu, displayed recognizing Members in the Personal Genome Paper by Name [9].Authors associated names and contact information to openly available profiles in the Personal Genome Paper. These profiles contain restorative and genomic information, including experiences about pharmaceuticals, strategies and ailments, and statistic data, for example, date of birth, sexual orientation, and postal code. By connecting socioeconomics to open records, for example, voter records, and digging for names covered up in joined reports, they accurately distinguished 84 to 97 percent of the profiles for which we gave names. Their capacity is to take in their names depends on their socioeconomics, not their DNA, in this way returning to an old weakness that could be effortlessly frustrated with negligible loss of research esteem. Along these lines, they propose specialized solutions for individuals to find out about their socioeconomics to settle on better choices.

E. S. Ackley and F. Esponda, proposed ensuring data security through difficult to invert negative databases [10].The paper expands negative portrayals of data for upgrading protection. Basically, a set DB of information components can be spoken to regarding its supplement set. That is, every one of the components not in DB are delineated and DB itself isn't unequivocally put away. Creators audit the negative database (NDB) portrayal plot for putting away a negative picture minimalistically and propose an outline for delineating a numerous record DB utilizing an accumulation of NDBs - as opposed to the single NDB approach of past work. At long last, they present a strategy for making negative databases that are difficult to turn around by and by, i.e., from which it is difficult to acquire DB, by adjusting a procedure for producing 3-SAT equation

\section{CONCLUSION}

Here, In this paper, we have done a sharing of individual genomic data without violating the protection of data to protect gigantic scale biomedical research meanders. We have taken the structure proposed by Kantarcioglu et al. In this context we have used homomorphic encryption technique and two servers. One holds the keys and another anchors the encoded records. The proposed framework has two new fixations in space time trade off and handles new sort of demands. Such that the strategy helps for upgrading of nucleotides which is sensible and central requirement of biomedical specialist. Huge information examination over hereditary information is a decent future work course. There are quick late kinds of advancement that address execution objectives of homomorphic encryption strategies. We accept that these degrees of advancement will incite more useful game plans later on that can manage greater scale inherited characteristics data. It justifies saying that our approach isn't 
bound to a settled homomorphic encryption methodology and in this way, it is possible to use and get the advantages of as of late made ones.

\section{REFERENCES}

1. M. Kantarcioglu, W. Jiang, Y. Liu, and B. Malin, “A cryptographic approach to securely share and query genomic sequences," Inf. Technol. Biomed. IEEE Trans., vol. 12, no. 5, pp. 606-617, 2008.

2. B. Malin and L. Sweeney, "How (not) to protect genomic data privacy in a distributed network: using trail re-identification to evaluate and design anonymity protection systems," J. Biomed. Inform vol. 37, no. 3, pp. 179-192, 2004.

3. E. Aguiar, Y. Zhang, and M. Blanton, "An Overview of Issues and Recent Developments in Cloud Computing and Storage Security," in High Performance Cloud Auditing and Applications, 2014, pp. 3-33.

4. P. Bohannon, M. Jakobsson, and S. Srikwan, "Cryptographic Approaches to Privacy in Forensic DNA Databases," in Public Key Cryptography, vol. 1751, H. Imai and Y. Zheng, Eds. Springer Berlin Heidelberg, 2000, pp. 373-390.

5. F. Bruekers, S. Katzenbeisser, K. Kursawe, and P. Tuyls, "Privacy-preserving matching of DNA profiles," IACR Cryptol. ePrint Arch., vol. 2008, p. 203, 2008.

6. M. Blanton and M. Aliasgari, "Secure outsourcing of DNA searching via finite automata," in Data and Applications Security and Privacy XXIV, Springer, 2010, pp. 49-64.

7. M. J. Atallah and J. Li, "Secure outsourcing of sequence comparisons,” Int. J. Inf. Secur., vol. 4, no. 4, pp. 277-287, Mar. 2005.

8. A. E. Nergiz, C. Clifton, and Q. M. Malluhi, "Updating outsourced anatomized private databases," in Proceedings of the 16th International Conference on Extending Database Technology, 2013, pp. 179-190.

9. L. Sweeney, A. Abu, and J. Winn, "Identifying Participants in the Personal Genome Paper by Name," Available SSRN 2257732, 2013.

10. F. Esponda, E. S. Ackley, P. Helman, H. Jia, and S. Forrest, "Protecting data privacy through hard-to-reverse negative databases,” Int. J. Inf. Secur., vol. 6, no. 6, pp. 403-415, 2007. 